\title{
Visual Communication and Heuristics: Challenges and Directions from Across the Disciplines
}

\author{
David M. Berube* \\ Dept. of Communication \\ North Carolina State University
}

\begin{abstract}
In communication scholarship attention is given to heuristics and mental shortcuts when designing persuasive messages of all sorts. We are closely aligned to cognitive psychology when considering the means of persuasion and argument. Whether selling consumer products or attempting to attract voters, how the public reads the messages we design is critically important. This brief paper examines some of the visual heuristic work that has been done and suggests we approach visual heuristics as a data-driven social science rather than an aesthetic exercise guide by intuition and experience.
\end{abstract}

Index Terms: Human-centered computing-Visualization-Visualization techniques-; Human-centered computing-Visualization-Visualization design and evaluation methods

\section{Mental Short Cuts}

The public are "cognitive misers" [9,20]: the tendency of people to think and solve problems in simpler and less effortful ways rather than in more sophisticated and more effortful ways, regardless of intelligence. As such, when confronted with information difficult to comprehend they tend to use "mental short cuts" known as heuristics [1]. Deferring to these mental short cuts they exhibit biases very often and draw wrong conclusions.

These "mental short cuts" are examined in the communication related literature to explain why some inexplicable conclusions are reached from public audiences, whether hearing or reading an argument, that are not compatible and often wholly contrary, to the purpose of the persuasive message. Witness conspiracy arguments.

At other times, these "mental short cuts" work well. They are built from experience and become the intuitive short-cuts audiences make when they confront similar challenges to their comprehension. Witness how people decide which movies to see.

Motivation for these mental short cuts are not wholly laziness or stupidity per se. The audience has many concepts fighting for attention some highly pragmatic such as how to feed a family of three or more with the primary earner on minimum wage employment or how to pay for mounting medical bills. The glut of information demanding their attention is proliferating $[14,18]$.

Other times, we have an audience composed of individuals who did not have the wherewithal to secure a high-quality education. And other times, we have individuals who have rejected developing the toolset needed to understand complex ideas in politics, in science, and in literature.

\section{Heuristics}

A way to understand heuristics in communication is to consider eye-tracking research where we find the public attends to different locations on a webpage [6-8]. Scholars and practitioners have used this literature and its conclusion to optimize web driven outreach and general communication.

*e-mail: dmberube@ncsu.edu
Generally, Western audiences focus on upwards and leftwards. Unsurprisingly, this can be associated with how we have been taught to read. When we open a book or a newspaper, we start there. When we open a webpage, we start there as well. This is a bias and it serves as short-cut toward understanding.

In the field of communication, we cover dozens or heuristics in persuasion studies. Some are very specific to print media, though the vast majority are not and surprisingly, how mental short cuts may impact visual messaging has received minor coverage in the field of visual communication. A good primer in the field of visual communication comes from Lester now in its 6th edition [16].

The primary crossover heuristic is likely to be innumeracy [12,19]. Innumeracy distorts risk perception towards health decisions and may negatively affect economic choices.

The second clear crossover is "probability neglect" and it is probably associated with innumeracy. When decision making under uncertainty, people regularly violate the normative rules for decision making whereby small risks are typically either neglected entirely or hugely overrated. The continuum between the extremes is ignored [13,21].

Another may be "base rate neglect" also associated with both above. "Base rates are largely ignored if individuating information is made available, which in turn drives people to respond stereotypically based on the "essential features of the evidence" [15,23].

Heuristics and mental short-cuts have found their way into many works in the design and computer aided communication. Graphic design scholars spend much of their time attempting to communication design literacy [11]

Discussions on heuristics can be discerned from some online survey work where we have rediscovered: recency and latency, midpoint represents typical, and spacing bias. From eye-scanning, we have developed a handful of literacy biases: left and top, near means related, up means good [22].

\section{Visual Aids and Literacy}

One of the crossover articles into communication has been evidencebased heuristics based on the design of visual aids, both static and interactive/dynamic and its association with populations with high or low numeracy and graphic literacy [10].

Discerning the assumptions and presuppositions associated with what separates the graphical literate and the graphical illiterate will help us to explain why some messages works for some subset of our audiences and not for others.

Assumedly, just like how science communication scholar discovered that the science deficit theory was not highly correlated to the public misunderstanding of science and send scientists into the public sphere to instruct the public was a dismal failure, supplementing the public with additional graphical and visual cues will probably yield similarly ineffectual results.

Avgerinou and Ericson attempted to wrestle with what constitutes visual literacy twenty years ago with mixed results [2]. You can always take a pricey Tufte course and come home with some thumb rules and pretty books. Visual literacy continues the tradition of Debes [3], Amheim [3], and Dondis [3]. Moore and Dwyer (1994) have advanced the legitimacy of visual messaging as a bona fide 
language, and have begun to answer many of the questions about visual literacy concepts; however, there remains a need to establish a common grammar for visual messages that is founded upon a definition of visual literacy [3].

Graphics literacy, a subset of visual literacy, is very important as well. From charts to graphs, diagrams to floor plans, layout of links from online search engines, understanding three-dimensional representations of proteins, how surfaces are decoded, etc. Graphical literacy scholars have faced similar challenges [4]. Efforts towards graphical literacy have taken many forms including in-classroom instruction in K-12, ACTs Workplace Graphics Literacy Test, etc.

Identifying what information is being presented and understanding how to use it may be critical to success in the 21 st century for a host of professions and for the general public to enjoy living in a digital world.

It must be clear to even the most cynical of us that communication as it interfaces closely with graphical and visual imagery is playing an ever-increasing role in the day to day life of a wider spectrum of society.

Results from a NAAL study found that 22 percent of American adults fall within the lowest level of quantitative literacy (i.e., below basic levels) [5]. When graphics as visual adjuncts are co-tested with and/or without traditional text, we find mixed results leading scholar to conclude that many people with low numeracy might still have difficulties interpreting and using numerical concepts when they are visually presented [10].

\section{Challenges}

We are confronted by quite a few challenges and stronger active relationships between the fields of communication, computer science, and design seems to be justified. We might want to begin with the following questions:

- What can we learn from both the disciplines of communication and design that will help explain why some populations do not readily benefit from graphical communication?

- What heuristics or short-cuts in understanding does the public use when confronted with complex information?

- Can we establish tools to evaluate the specific heuristically driven trials we need to engage and resolve to maximize efforts at communicating meaning?

- Can we produce data-driven findings that reach beyond traditionally text-based communication through digital-based developments to those of graphics and design?

New insights into the heuristics of communication, textual and visual, may provide us with new tools to make messages more understandable and effective. Setting disciplinary barriers aside, where they exist, and embracing the new convergence riding the digital tsunami of platforms, procedures, and processes, we have a lot to share and lot of learn.

Additional reading: a typical communication text on applied visual communication [17].

\section{ACKNOWLEDGMENTS}

This work was supported in part by NSF ECCS 1542015; NNCI: North Carolina Research Triangle Nanotechnology Network (RTNN).

\section{REFERENCES}

[1] M. Andrejevic. Infoglut: How too much information is changing the way we think and know. Routledge, 2013.

[2] M. Avgerinou and J. Ericson. A review of the concept of visual literacy. British Journal of Educational Technology, 28(4):280-291, 1997.
[3] J. M. Brill and R. Maribe Branch. Visual literacy defined-the results of a delphi study: can ivla (operationally) define visual literacy? Journal of Visual Literacy, 27(1):47-60, 2007.

[4] J. Debes. Some foundations for visual literacy. Audiovisual instruction, 13(9):961-964, 1968.

[5] D. A. Dondis. A primer of visual literacy. Mit Press, 1973.

[6] M. K. Eckstein, B. Guerra-Carrillo, A. T. M. Singley, and S. A. Bunge. Beyond eye gaze: What else can eyetracking reveal about cognition and cognitive development? Developmental cognitive neuroscience, 25:69-91, 2017.

[7] L. Eger. How people acquire knowledge from a web page: An eye tracking study. Knowledge Management \& E-Learning: An International Journal, 10(3):350-366, 2018.

[8] S. Eraslan, Y. Yesilada, and S. Harper. Scanpath trend analysis on web pages: Clustering eye tracking scanpaths. ACM Transactions on the Web (TWEB), 10(4):20, 2016.

[9] S. T. Fiske and S. E. Taylor. Social cognition: From brains to culture. Sage, 2013.

[10] R. Garcia-Retamero and E. T. Cokely. Designing visual aids that promote risk literacy: A systematic review of health research and evidence-based design heuristics. Human factors, 59(4):582-627, 2017.

[11] S. Heller. Design literacy: Understanding graphic design. Simon and Schuster, 2004.

[12] D. R. Hofstadter. Metamagical themas. Scientific American, 246(5):20D, 1982.

[13] D. Kahneman. Thinking, fast and slow. Macmillan, 2011.

[14] D. Kahneman, S. P. Slovic, P. Slovic, and A. Tversky. Judgment under uncertainty: Heuristics and biases. Cambridge university press, 1982.

[15] D. Kahneman and A. Tversky. On the psychology of prediction. Psychological review, 80(4):237, 1973.

[16] P. M. Lester. Visual communication: Images with messages. Cengage Learning, 2013.

[17] D. M. Moore. Visual literacy: A spectrum of visual learning. Educational Technology, 1994.

[18] L. V. Orman. Information Overload Paradox: Drowning in Information, Starving for Knowledge. 2016.

[19] J. A. Paulos. Innumeracy: Mathematical illiteracy and its consequences. Macmillan, 1988.

[20] K. E. Stanovich. What intelligence tests miss: The psychology of rational thought. Yale University Press, 2009.

[21] C. R. Sunstein. Probability neglect: Emotions, worst cases, and law. Yale $L j, 112: 61,2002$.

[22] V. Toepoel and D. A. Dillman. Words, numbers, and visual heuristics in web surveys: Is there a hierarchy of importance? Social Science Computer Review, 29(2):193-207, 2011.

[23] O. Vartanian, E. L. Beatty, I. Smith, K. Blackler, Q. Lam, S. Forbes, and W. De Neys. The reflective mind: Examining individual differences in susceptibility to base rate neglect with fmri. Journal of cognitive neuroscience, 30(7):1011-1022, 2018. 